\title{
Les écrits du « for privé » du clergé émigré
}

The Writings of the "for privé" of the Emigre Clergy

\section{Stéphane Gomis}

\section{(2) OpenEdition}

\section{Journals}

\section{Édition électronique}

URL : https://journals.openedition.org/ahrf/10749

DOI : $10.4000 /$ ahrf. 10749

ISSN : 1952-403X

Éditeur :

Armand Colin, Société des études robespierristes

Édition imprimée

Date de publication : 1 janvier 2009

Pagination : 183-204

ISBN : 978-2-200-92557-4

ISSN : 0003-4436

\section{Référence électronique}

Stéphane Gomis, «Les écrits du «for privé » du clergé émigré », Annales historiques de la Révolution française [En ligne], 355 | janvier-mars 2009, mis en ligne le 01 janvier 2012, consulté le 22 avril 2022. URL : http://journals.openedition.org/ahrf/10749 ; DOI : https://doi.org/10.4000/ahrf.10749 


\title{
LES ÉCRITS DU «FOR PRIVÉ » DU CLERGÉ ÉMIGRÉ
}

Stéphane GOMIS

\begin{abstract}
L'étude des manuscrits du "for privé » permet d'approcher cette dimension essentielle qui touche au vécu des individus. Ces écrits fournissent l'occasion de mieux comprendre les mentalités à l'œuvre à une période donnée, en l'occurrence en cette fin du XVIII ${ }^{e}$ siècle. Ce sont trente-six témoignages émanant de gens d'Église issus du clergé séculier qui ont pu être rassemblés. En définitive, ces documents témoignent d'aventures personnelles. En cela, le monde des émigrés apparaît donc dans toute sa diversité et sa complexité. Cependant, ils permettent d'éclairer le devenir d'un groupe en particulier. Longtemps considérés, soit comme des martyrs, soit comme de dangereux contre-révolutionnaires, ces clercs apparaissent tout d'abord comme des hommes qui ressentent des émotions souvent contradictoires. Pour l'essentiel, ils ont cherché surtout à survivre dans un environnement étranger et parfois hostile. Mais l'exil leur a donné également l'occasion de se confronter à l'altérité.
\end{abstract}

Mots-clés : Clergé, émigration, « for privé », religion, livre de comptes.

Abrégé ou relation sommaire des lugubres événements de la Révolution française. En particulier, exposé succinct des faits qui me concerne personnellement, moi, P.-C. Blanckaert, curé de Wormhoudt et doyen de Chrétienté à cette époque de malheur ${ }^{1}$. Tel est le titre exact donné par Pierre-Corneille Blanckaert (1724-1799), curé de la paroisse de Wormhoudt

(1) « Le manuscrit de M. P.-C. Blanckaert, curé-doyen de Wormhoudt, traduit du flamand par M. l'abbé L. Harrau et Ed. Blomme », Bulletin de l'Union Faulconnier (Dunkerque), t. V, 1902, p. 191-259. 
dans le diocèse d'Ypres, à ses remarques consignées au jour le jour par ce prêtre parti en 1792, mort à Bruges en 1799. "Lugubres événements », « époque de malheur », voilà les termes utilisés par ce curé pour qualifier son temps. À la lecture de tels qualificatifs, l'historien aurait pu arrêter là ses investigations sur les écrits du « for privé » d'hommes, dont on pourrait présager à l'évidence que leurs témoignages sont à ranger parmi toute une littérature contre-révolutionnaire. De fait, pendant longtemps, l'étude de l'émigration ecclésiastique a été l'apanage d'auteurs, pour la plupart issus eux-mêmes du clergé, dont le principal objectif était de célébrer le culte de ces « martyrs » de la Révolution contraints à l'exil'. Pourtant, si le sujet n'a guère obtenu la faveur de l'historiographie la plus récente, quelques travaux sont venus renouveler notre approche ${ }^{3}$. Cette contribution n'entend pas répondre à toutes les questions suscitées par ce type de source. Il s'agit, plus modestement, d'ébaucher quelques résultats d'une enquête sans doute bien imparfaite. Ainsi, les écrits du for privé ne sont-ils pas de nature à nuancer, à infléchir sur certains points en tout cas, notre connaissance du monde des clercs émigrés ? Quels sont les apports et les limites de ces documents? Précisons que notre étude porte exclusivement sur le clergé catholique séculier. Tout d'abord, il a semblé indispensable de définir

(2) Voir notamment, Abbé Hubert MaILfait, La déportation et l'exil du clergé français pendant la Révolution, Paris, Bloud, 1905 et Abbé Amans-Claude SABATIÉ, La déportation révolutionnaire du clergé français, Paris, J. Gabalda, 1916, 2 vol.

(3) René Picheloup, Les ecclésiastiques français émigrés ou déportés dans l'État pontifical, 1792-1800, Toulouse, Publication de 1'Université de Toulouse-Le Mirail, 1972 ; Dominic Aidan BeLLENGER, The French Exiled Clergy in the British Isles after 1789. An Historical Introduction and Working List, Bath, Downside Abbey, 1986 et, tout dernièrement, Manuel Gutiérrez GARCIA-BrazALES, El exilo del Clero Francés en España durante la Revolución (1791-1815), Zaragoza, 2004 et Bernward KRöGER, Der französische Exioiklerus im Fürstbistum Münster (1794-1802), Mayence, Verlag Philipp von Zabern, 2005 (Merci à Karine Rance pour cette dernière référence). D'autres auteurs ont également consacré des développements spécifiques à cette question comme Georges ANDREY, Les émigrés français dans le canton de Fribourg (1789-1815). Effectifs, activités, Portraits, Neuchâtel, Éditions de la Baconnière, 1972 ou encore de façon plus synthétique, Paul CHRISTOPHE, 1789, les prêtres dans la Révolution, Paris, Éditions ouvrières, 1986 et Charles CHAuvin, Le clergé à l'épreuve de la Révolution (1789-1799), Paris, Desclée de Brouwer, 1989. Pour être tout à fait précis, l'historien Victor Pierre a été l'un des tous premiers, au tournant des XIX et XX ${ }^{\mathrm{e}}$ siècles, à attirer l'attention des chercheurs sur cet aspect de l'émigration, auquel il a consacré plusieurs articles : « L'abbé de Montrichard et l'émigration française à Fribourg », Revue des questions historiques, t. 15, 1896, p. 142-167; «Le clergé français en Allemagne pendant la Révolution », Revue des questions historiques, t. 19, 1898, p. 148-184 ; « Le clergé français en Savoie et en Piémont, d'après les souvenirs inédits du chanoine Berlioz, 1791-1794 », Revue des questions historiques, t. 20, 1898, p. 119-158; « Le clergé français en Angleterre (17911802) », Revue des questions historiques, t. 22, 1899, p. 426-480 ; «Un curé de Normandie, Pierre Goudemetz, curé de Crétot, réfugié en Angleterre (1792-1801), d'après sa correspondance inédite », Revue des questions historiques, t. 24, 1900, p. 472-510; « Le clergé français dans les États pontificaux (1789-1803) », Revue des questions historiques, t. 27, 1902, p. 103-143, et enfin, « Le clergé français en Espagne 1791-1802 », Revue des questions historiques, t. 31, 1904, p. 473-538. 
précisément les caractéristiques du corpus étudié. Ensuite, nous porterons notre regard sur les considérations matérielles et les questions religieuses ; puis, nous nous interrogerons sur la nature des opinions portées par les scripteurs sur le champ politique, mais également, sur les mœurs des pays d'accueil $1^{4}$.

\section{De la nature du « for privé »}

« La vie privée comme la vie intime ne sont pas aisées à atteindre, soit qu'elles se confondent avec la vie publique, soit qu'elles aient été cachées aux autres par la réserve même à les révéler ». C'est en ces termes que Madeleine Foisil introduit le chapitre consacré, au sein de l'Histoire de la vie privée, à "l'écriture du for privé $»^{5}$. En effet, l'historien est parfois bien en peine de sonder les reins et les cœurs. Il lui est souvent difficile de pénétrer dans l'intimité d'hommes, pourtant au centre de ses recherches. Or, l'étude des manuscrits du for privé, appelés également egodocuments, permet d'approcher cette dimension essentielle qui touche au vécu des individus. Ces témoignages fournissent l'occasion de mieux comprendre les mentalités à une période donnée, en l'occurrence en cette fin du XVIII siècle. Les écrits du for privé désignent l'ensemble des documents qui impliquent la prise de parole personnelle d'un ou plusieurs individus. Ils sont de différentes natures. Une typologie rapide distingue d'une part, le « livre de raison » sur lequel le père de famille consigne les différents événements familiaux ou bien les comptes du ménage, mais également des remarques plus personnelles; d'autre part la « chronique » davantage orientée sur le récit de la vie locale; enfin, le journal, depuis le « diaire », tenu au jour le jour, jusqu'au « journal de voyage ». Mais, en définitive, un manuscrit peut relever de plusieurs genres différents.

(4) Considérant la littérature de l'émigration française pendant la Révolution, Bernard Plongeron a écrit que les « Journaux, carnets, mémoires... ont généralement un caractère d'autant plus anecdotique qu'ils sont fragmentaires dans l'espace et dans la durée » ("Église et Révolution, d'après les prêtres émigrés à Rome et à Londres (1792-1802) », Christianesimo nelle storia, $\mathrm{n}^{\circ} 10$, 1989 , p. 273). Sans nier la dimension de cette source, nous nous proposons ici de montrer qu'il est possible de dépasser ces aspects anecdotiques, afin de dégager certains traits communs. Pour ce qui concerne les mémoires de la noblesse, l'historiographie a été largement renouvelée notamment par la thèse de Karine RANCE, Mémoires de nobles émigrés dans les pays germaniques pendant la Révolution française, Thèse de doctorat, Université Paris 1, 2001. Voir également, du même auteur, " Mémoires de nobles français émigrés en Allemagne pendant la Révolution française : la vision rétrospective d'une expérience», Revue d'histoire moderne et contemporaine, t. 46, 1999, p. 245-262.

(5) Philippe Ariès et Georges Duby (dir.), Histoire de la vie privée (tome 3). De la Renaissance aux Lumières, Paris, Le Seuil (Points Histoire), 1999 ( $1^{\text {re }}$ édition 1985), p. 319-357. 
Plusieurs aspects se mêlent souvent les uns aux autres ${ }^{6}$. Nous nous intéresserons ici au journal, la plupart du temps saisi sur le vif ou à partir de notes recueillies a posteriori, par des individus partis de leur pays natal pour trouver refuge en des contrées généralement inconnues jusqu'alors?

La première étape a donc consisté à réaliser un recensement des écrits du for privé émanant de gens d'Église issus du clergé séculier. Lors d'une étape préalable, il importait de s'appuyer sur les travaux de recension parus essentiellement à la faveur de la célébration du Bicentenaire, tel que celui d'Alfred Fierro ${ }^{8}$. Il s'agissait ensuite de compléter cet inventaire, déjà très riche, notamment en repérant les écrits publiés, fut-ce de façon incomplète, depuis la fin des années 1980. Pour ce faire, nous nous sommes appuyés sur plusieurs instruments de recherches. Le Catalogue collectif de France, ainsi que celui de la Bibliothèque nationale, ont fourni d'utiles références. Au total, ce sont 36 publications qui ont pu être rassemblées. Il convient de préciser que ce travail ne prétend pas être exhaustif. Sur cet ensemble, dix-sept auteurs étaient curés. Trois occupaient les fonctions de vicaire. Trois avaient la qualité de chanoine. Pour leur part, Jean Brumauld de Beauregard (1749-1841) et François Le Noir de La Cochetière (1755-1828) étaient vicaires généraux, le premier du diocèse de Luçon, le second de celui d'Angers. Henri-Alexandre Boissière (1745-1805) et Antoine Vieux (1752-1816) étaient pour l'un secrétaire de l'évêque de Quimper, pour l'autre, chapelain de l'Hôtel-Dieu de Lyon. Quant à François Delestre (1761-1798), il était professeur dans un collège à Neuchâtel-en-Bray. Enfin, le statut exact de huit scripteurs n'a pu être déterminé. Cependant, la mention «prêtre » accolée au nom de l'auteur permet de les rattacher indubitablement à notre corpus.

(6) Sylvie Mouysset, Papiers de famille. Introduction à l'étude des livres de raison (France, $X V^{e}-X I X^{e}$ siècle), Rennes, PUR, 2008.

(7) Jusqu'à présent ce type de source a essentiellement été utilisé pour illustrer tel ou tel aspect de la vie des émigrés, sans toujours prendre en compte les spécificités de l'émigration ecclésiastique. Voir Duc de CAstries, La vie quotidienne des émigrés, Paris, Hachette, 1966 et Ghislain de Diesbach, Histoire de l'émigration 1789-1814, Paris, Perrin, 1998. L'étude de certaines sources archivistiques, telles que la correspondance « des Messieurs des Missions Étrangères de Paris », a connu un meilleur sort, voir B. Plongeron, «Église et Révolution, d'après les prêtres émigrés à Rome et à Londres (1792-1802)» [...], p. 273-305. Voir également, du même auteur, Conscience religieuse en Révolution. Regards sur l'historiographie religieuse de la Révolution française, Paris, Picard, 1969.

(8) A. Fierro, Bibliographie critique des mémoires sur la Révolution écrits ou traduits en français, Paris, Service des travaux historiques de la ville de Paris, 1988. 
Au regard du total de prêtres ayant quitté le territoire national, ce chiffre semble bien mince. En effet, le nombre de prêtres et de religieux partis en exil approche vraisemblablement les 30000 individus9. Autrement dit, un quart environ des effectifs du premier ordre du royaume présents en 1789. Si nous pouvons émettre l'hypothèse que certains témoignages ont été perdus ou bien que d'autres n'ont pas encore été exhumés et publiés, les découvertes futures ne bouleverseront sans doute pas de façon significative ce chiffre ${ }^{10}$. Par ailleurs, ce faible corpus rappelle à l'évidence que la prise de parole à travers la rédaction de ces notes de voyage est une décision purement personnelle. Elle émane avant tout de l'individu qui entend se rendre compte à lui-même du chemin parcouru, des émotions ressenties ou bien, plus prosaïquement, des dépenses engagées. Il s'agit donc, par essence, d'une décision dictée par la seule volonté de son auteur. Il écrit donc avant tout pour lui-même. Par ailleurs, il apparaît évident que ces témoignages n'étaient pas destinés à être connus du public. Ainsi, les dates de leur publication indiquent suffisamment qu'ils n'avaient pas vocation à être diffusés. Tous ont été dévoilés après la mort des protagonistes. La publication de la prose de François Delestre (17611798), survenue en 1819 , soit une vingtaine d'années après sa mort, fait figure d'exception. En effet, la majorité des témoignages a été exhumée entre les années 1880 et 1910. L'initiative en revenait souvent à des clercs qui trouvaient là l'occasion de condamner une Révolution qui avait jeté sur les routes un clergé qui s'était refusé à servir le « schisme ». L'époque de la célébration du premier centenaire de la Révolution et les événements survenus au moment des lois de séparation de l'Église et de l'État

(9) Les travaux les plus récents, qui se sont attachés à quantifier ces départs, s'accordent désormais sur ce chiffre. Bernard Plongeron évoque même la fourchette de 25 à 30000 individus, après avoir indiqué que de nombreuses publications, "sur la foi d'estimations fort anciennes », ont majoré «l'ampleur des mouvements de l'émigration ecclésiastique », allant jusqu'à retenir une fourchette comprise entre 40 et 60000 personnes (voir sa contribution sur la « Géographie de l'émigration ecclésiastique » dans Jean-Marie Mayeur, Charles et Luce Pietri, André Vauchez et Marc Venard, Histoire du Christianisme, (tome X). Les défis de la modernité, Paris, Desclée de Brouwer, 1997, p. 411-416). Le chiffre de 30000 est également avancé par Charles Chauvin. Cependant, l'addition du décompte qu'il fournit pour chaque pays donne celui de 28100 (Le clergé [...], p. 91-97).

(10) Il est utile de préciser que nous n'avons pas eu le temps de nous engager dans une quête des manuscrits conservés dans les bibliothèques ou les dépôts d'archives. Cependant, les ressources fournies par les bases de données, déjà considérables, constituées par le GDR (CNRS) n 2649 consacré aux « Écrits du “for privé” du Moyen Âge à 1914 » n'indiquent aucun document de cette nature. À ce jour, seul le « livre de comptes » de l'abbé Nicolas Marnat, que j'ai découvert récemment aux archives départementales du Puy-de-Dôme, vient contredire cette assertion. Il sera évoqué infra. 
constituent en l'occurrence des temps forts. En 1867 déjà, en publiant le journal du curé auvergnat Paul Valeix (1739-1827), l'abbé JacquesBlaise Danglard écrit : "C'est la mémoire d'un confesseur de la foi qu'il nous est donné de perpétuer ». Il entend également « raviver dans quelques âmes la force chrétienne par la vue des épreuves et de la généreuse conduite d'un saint prêtre $\rangle^{11}$. L'expression "prêtre déporté » utilisé par ce clerc éditeur, mais également par d'autres érudits, commande de s'arrêter quelques instants sur la terminologie employée pour désigner ces migrations cléricales. Doit-on parler d' "émigration », de "proscription» ou encore de "déportation»? Comme l'indiquent justement les auteurs de l'Atlas de la Révolution française, en la matière, 《le vocabulaire reste hésitant $»^{12}$. En définitive, ces termes renvoient à la fois à des situations particulières et à une législation qu'il est nécessaire de rappeler.

En effet, peu nombreux sont les membres du clergé qui décident d'émigrer dès 1789. En 1791, après la crise consécutive au serment à la Constitution civile du clergé, plusieurs évêques s'engagent dans la voie de l'émigration. À cette époque, seuls quelques autres clercs participent à ce mouvement, à l'image du religieux prémontré Hervé-Julien Le Sage (1757-1832) qui, en juillet 1791, s'embarque pour l'île de Jersey, avant de gagner l'Angleterre. Il rejoint ensuite le continent pour un périple qui le conduira en Silésie, en passant par les terres allemandes et l'Italie ${ }^{13}$. Il s'agit donc d'une émigration qui ne s'effectue pas sous la contrainte pressante d'un texte de loi. Tel n'est pas le cas de l'exil massif qui touche les clercs, à la suite du décret du 26 août $1792^{14}$. Ce dernier fait obligation aux prêtres insermentés de moins de 60 ans, de quitter dans les quinze jours le territoire national, sous peine de déportation ${ }^{15}$. En l'espace de quelques semaines, ce sont donc des milliers d'hommes qui vont franchir les frontières. Il s'agit bien d'une proscription dans le sens d'une émigration commandée par des

(11) Abbé B.-J. Danglard, De Clermont à Genève. Journal d'un prêtre déporté en 1792, Clermont, F. Thibaud, 1867.

(12) Serge Bonin et Claude Langlois (dir.), Atlas de la Révolution française (tome 9). Religion, Paris, EHESS, 1996, p. 36.

(13) Xavier Lavagne d’Ortigue, De la Bretagne à la Silésie. Mémoires d'exil de HervéJulien Le Sage (1791 à 1800), Paris, Beauchesne, 1983.

(14) Quelques éléments récents sur le mouvement quotidien des demandes de passeports auprès de la municipalité de Lyon pour les mois d'août et de septembre 1792, dans Paul CHOPELIN, Ville patriote et ville martyre. Une histoire religieuse de Lyon pendant la Révolution (1788-1805), Thèse de doctorat, Université Jean Moulin-Lyon III, vol. 1, p. 334-337.

(15) Cette législation est parfaitement bien exposée par Jacques Godechot, La ContreRévolution : doctrine et action, 1789-1804, Paris, PUF, 1984, p. 155-156. 
obligations légales. On aura noté que le terme de déportation est employé par le législateur comme condamnation appliquée aux contrevenants. En définitive, les événements survenus dans les départements de la Sarthe et $\mathrm{du}$ Maine-et-Loire montrent que des réfractaires furent effectivement déportés, dans le cas présent par anticipation. Dès le 28 août, le directoire départemental décide que 146 réfractaires détenus au séminaire du Mans seraient conduits à Nantes, afin d'y être embarqués. En passant par Angers, 264 autres prêtres viennent grossir les rangs des proscrits. Enfin, après avoir été emprisonnés quelques temps au château de Nantes, les condamnés sont répartis entre trois navires qui, après une traversée mouvementée, finissent par aborder les côtes espagnoles ${ }^{16}$. Embarqué sur La Didon, Jacques Peccard (1755-1837), vicaire de la paroisse angevine Saint-Aubin de Pouancé, conte combien ce voyage de huit jours ne fut pas aisé, avant qu'il ne s'achève dans le port de Santander le 11 octobre $^{17}$.

En quittant leur terre natale, ces voyageurs ignorent bien souvent quels seront le lieu et le temps de leur exil. Sentiment bien compréhensible, ces exilés espéraient que leur séjour contraint serait le plus bref possible. Pourtant, en moyenne, il va s'échelonner sur une dizaine d'années. Les retours les plus importants ont lieu au moment de la ratification du Concordat en 1802. «Les terres d'asile sont commandées surtout par la géographie $»^{18}$. Dans un premier temps, il est vrai que le clergé, pour des raisons d'accessibilité plus facile, se dirige prioritairement vers des pays proches. Les Normands et une partie des Bretons se réfugient majoritairement en Angleterre. Les Francs-Comtois ou les Bourguignons se retrouvent en grand nombre dans le canton suisse de Fribourg, tandis que les Dauphinois ou les Provençaux prennent le chemin des États pontificaux. Certains se fixent tout de suite en un endroit, notamment parce que des dispositions propres à chaque nation les obligent à fixer leur résidence en un lieu bien spécifique. D'autres entament un véritable périple à travers l'Europe toute entière. En réalité, ces exilés sont rarement maîtres de leur choix. Des contraintes diverses, liées notamment à la progression des armées révolutionnaires, les conduisent trop souvent à leur goût à changer le lieu de leur résidence. Les nécessités matérielles, qu'accompagne

(16) Pierre de LA Gorce, Histoire religieuse de la Révolution française, Paris, Plon, 1912, t. 2, p. 297-301.

(17) Ce manuscrit dont l'intitulé est le suivant : «Écrit en Espagne, au bourg de Somahoz, vallée de Buelna, par moi J. Peccard, en 1793 », a été édité accompagné de quelques notes par l'abbé Aimé Lefort (M. Jacques Peccard 1755-1837. Histoire d'un prêtre déporté en Espagne pendant la Révolution française, Angers, Grassin, 1914).

(18) S. Bonin et C. Langlois (dir.), Atlas de la Révolution française [...], p. 37. 
parfois l'espoir d'une vie meilleure, les conduisent également à reprendre la route. Cette errance se décline à des degrés très divers.

Pour ceux dont le pays d'adoption est l'Espagne ou les États pontificaux, la législation témoigne d'une suspicion certaine à leur égard. En Espagne, les émigrés doivent prêter un serment de fidélité au roi, assorti de l'obligation de « ne pas parler de ce qui se passe dans leur pays ». Une cédule, en date du 2 novembre 1792 organise leur « confinement dans les couvents, leur interdit le logement chez des particuliers, ainsi que toute activité de prêche, de confession et d'enseignement $»^{19}$. Néanmoins, cette politique est un échec. Au mieux $40 \%$ du clergé peut être accueilli dans un établissement religieux. À cet égard, le destin de Jacques Peccard donne l'exemple de l'incapacité des autorités espagnoles à appliquer rigoureusement ce texte. Certes, dès le lendemain de son arrivée il est convoqué, avec ses compagnons de route, «à la maison de ville » où on leur fait prêter serment de fidélité à Charles IV. Ensuite, l'abbé Peccard ne rejoint pas un couvent mais se trouve hébergé chez un habitant de la ville «M. Redonet», négociant. Puis, le 17 décembre, il obtient la desserte d'une chapelle à Viono, bourg situé à une vingtaine de kilomètres de Santander, avant de desservir la paroisse proche de Somahoz en tant que vicaire, jusqu'à son départ en 1802. Tout au long de ces dix ans, Jacques Peccard fait donc preuve d'une stabilité tout à fait remarquable. À l'inverse, d'autres clercs vont connaître un nomadisme plus ou moins prononcé. Parti dès le 4 mai 1792 afin de se « mettre à l'abri de tout danger », Pierre-Corneille Blanckaert quitte la France. L'espace d'une année, il réside non loin de la frontière, tout d'abord à Poperinghe pendant huit mois, puis à Nieuwkerke durant quatre mois. Contraint de fuir devant l'avancée des troupes républicaines, il séjourne à Ypres puis à Zarven jusqu'au mois de juin 1794. À cette date, il entame un voyage qui, dans ses limites les plus orientales, va le conduire le 16 février 1795 jusqu'à Altenberghe, près de Munster. Sur le chemin, il parvient toutefois à se

(19) Jean-Philippe LuIs, « Une histoire de réfugiés politiques : le clergé français émigré en Espagne pendant la Révolution française », dans Anne DuBET et Stéphanie Urdician (études rassemblées par), Exils, passages et transitions. Hommage à Rose Duroux. Chemins d'une recherche sur les images, Clermont-Ferrand, Presses Universitaires Blaise Pascal, 2008, p. 25-34. Je remercie l'auteur de m'avoir signalé cette publication récente. J.-P. Luis est également l'auteur de l'article suivant : " Le clergé français émigré en Espagne pendant la Révolution (1791-1802) », dans Les révolutions ibériques et ibéro-américaines à l'aube du XIX siècle, Paris, CNRS, 1991, p. 45-58. Pour l'Espagne, la première étude sérieuse est celle de l'abbé Jean CONTRASTY, Le clergé français exilé en Espagne (1792-1802), Toulouse, L. Sistac, 1910. Si ce prêtre a travaillé dans un esprit contre-révolutionnaire, il a été le premier à s'intéresser aux sources espagnoles. 
ménager deux haltes. La première à Gemerkt en Brabant dure un mois, la seconde quatre mois à Werth. Le 25 août de la même année, ayant appris "que nombre de réfugiés retournaient en Flandre et en Brabant» et " qu'ils y étaient accueillis avec bienveillance et en toute sécurité », le curé de Wormhoudt s'engage dans un voyage de retour. Arrivé à Oostkerke, près de Bruges, au début du mois d'octobre 1795, il y séjourne jusqu'en février 1798, époque où il gagne Bruges jusqu'à sa mort survenue le 19 mai 1799. Pierre-Corneille Blanckaert offre l'exemple d'une mobilité que l'on pourrait qualifier de moyenne. Ce qualificatif s'explique pour deux raisons. La première est facteur de la distance parcourue. S'il reste difficile de mesurer précisément le nombre de kilomètres effectués, on peut toutefois estimer qu'il est proche de 500. Certes, le chiffre n'est pas négligeable, d'autant plus lorsqu'il faut envisager le trajet du retour et qu'il s'effectue à pied le plus souvent. Néanmoins, en comparaison avec des périples qui engagent leurs acteurs dans l'Europe toute entière, comme cela sera évoqué ultérieurement, les voyages du curé de Wormhoudt n'ont rien d'exceptionnel. La seconde est celle du temps de résidence passé dans certains lieux. En l'espace de sept ans, notre voyageur est tout de même parvenu à se ménager de longues plages de stabilitée ${ }^{20}$.

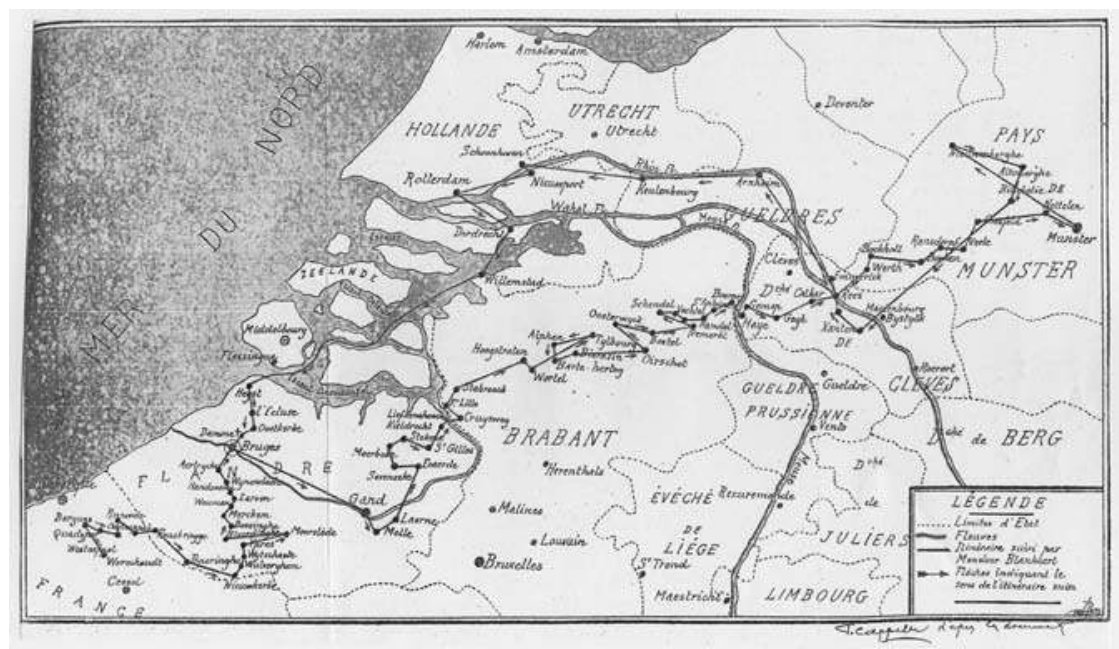

(20) La carte reproduite ici permet de visualiser l'ensemble du parcours suivi par PierreCorneille Blanckaert. Elle accompagne la publication de ses écrits. 
Toute autre est l'itinérance attachée au Normand Honoré Pétel (1744-1816), curé de Saint-Léger-du-Boscdel. On peut qualifier d'extrême sa mobilité, non seulement au regard des milliers de kilomètres parcourus, mais également en prenant en compte le temps mis pour gagner l'Italie depuis l'Angleterre, en passant par les États allemands et la Suisse ${ }^{21}$. Parti de Normandie, par le port de Dieppe, le 10 septembre 1792, il se fixe à Londres puis à Douvres. Après vingt mois de séjour outre-Manche, Pétel s'embarque le 19 avril 1794 pour la Flandre pour une halte de deux mois à Stekene. Il enchaîne alors un long voyage qui le conduit en novembre de la même année à Spalt, au sud de Nuremberg, où il reste sept mois. Le 18 juillet 1795, il est à Constance, où il se déclare «fatigué de courir ». Le curé normand reste dans cette ville jusqu'en décembre. Puis, dans les premiers jours de janvier 1796, sur les instances de l'évêque de Langres, César-Guillaume de la Luzerne (1738-1821), il gagne Vicence en Italie. Cette cité est le dernier lieu de sa résidence à l'étranger, qu'il quitte le 2 avril 1797 pour un nouveau périple de six semaines, via la Suisse et le Jura, qui le ramène dans sa province natale le 15 mai. Le grand tour européen effectué est donc tout à fait considérable. Il l'est d'autant plus que l'abbé Pétel traverse la Flandre, les Pays-Bas, puis l'Allemagne du nord au sud, en un peu plus d'une année seulement. Par ailleurs, si ce n'est en Angleterre et en Italie, il ne s'attache guère longtemps en des endroits en particulier.

\section{Des considérations profanes et sacrées}

À l'évidence, les moyens de subsistance constituent une préoccupation quotidienne des émigrés. Les aspects matériels sont donc très présents dans les récits de ces clercs. Privés de revenus, ayant rapidement épuisé les ressources financières dont ils avaient pu se munir, ces derniers se voient dans l'obligation de faire appel à la générosité d'autrui. Ces considérations participent pleinement de l'image du proscrit réduit à la plus extrême pauvreté. Elles renforcent la dimension du prêtre martyr. En effet, celui qui « professe le désagréable métier d'émigré » doit se procurer

(21) Charles Leroy, Sur les routes de l'exil (septembre 1792-mai 1797). Mémoires de M. l'abbé Sébastien-Jean-Honoré Pétel, curé de Saint-Léger-du-Boscdel (doyenné de Bernay), Rouen, Lestringant, 1929. Ce manuscrit a également été publié sous le titre suivant : "Voyages d'un prêtre déporté en $1792 »$, Revue rétrospective. Recueil de pièces intéressantes, $1888, \mathrm{n}^{\circ} 93$, p. 193-216; n 95, p. 241-264; n 104, p. 169-192 et n 106, p. 217-232. Il avait alors été attribué à l'abbé Renaud. En fait, celui-ci est Pierre-Modeste Regnault, l'un des compagnons du curé Pétel, également accompagné de ses deux frères, tous deux prêtres. 
le pain quotidien ${ }^{22}$. En l'espèce, il convient de distinguer deux cas de figure. L'un fait référence aux réseaux d'entraide qui permettent d'organiser la vie matérielle, mais également au système mis en place par les différents gouvernements, à travers l'hébergement dans quelques établissements religieux. L'autre est beaucoup plus empirique. Il s'appuie, dans le meilleur des cas, sur la charité octroyée par des institutions ou des particuliers, mais aussi sur les propres ressources des individus, à travers leur capacité à occuper divers emplois.

La première situation est particulièrement bien illustrée en Angleterre où le gouvernement, conduit par William Pitt, obtient du Parlement le vote d'une subvention annuelle en faveur des émigrés. Ces subsides sont répartis par l'évêque de Saint-Pol-de-Léon, Jean-François de la Marche (1729-1806). Par ailleurs, avec le concours principalement d'un autre prêtre breton, Guy Carron (1760-1821) et d'une anglaise, Dorothée Silburne, il organise un comité de secours, comprenant entre autres des centres d'hospitalisation et d'assistance. Dans les couvents espagnols ou italiens, le gîte et le couvert sont assurés, moyennant quelques conventions, comme nous le verrons plus loin. En Allemagne ou encore en Suisse, certains réseaux d'aide voient le jour, sans toutefois atteindre le degré d'organisation signalé en Angleterre. À Constance, une Mittagstaffel est organisée pour les ecclésiastiques nécessiteux afin de les nourrir, éventuellement de les vêtir, mais aussi de leur fournir les moyens nécessaires pour accéder aux soins médicaux ${ }^{23}$. Par ailleurs, les monastères ou les couvents constituent un maillage serré d'établissements susceptibles d'assurer l'accueil de ces pèlerins malgré eux. L'universalité de l'Église joue donc un rôle essentiel. Dans le voyage qui, entre le $1^{\text {er }}$ et le 18 juillet 1795, les conduit depuis Spalt jusqu'à Constance, Honoré Pétel et ses compagnons sont reçus systématiquement chez des réguliers. La palette des ordres représentés, qu'ils soient masculins ou féminins, est extrêmement large. Ce sont les bénédictins à Ehling ou à Wiblingen, les cisterciens à Kaiserheim, les dominicains à Medlingen, les cordeliers à Uberlingen, les capucins de Dillingen ou de Moeskirch. Les femmes sont représentées par les cisterciennes d'Helligkreustad ou de Clausterwald. La générosité des particuliers n'est également pas absente. Arrivé en janvier 1793 à

(22) P. de LA Gorce, Histoire religieuse [...], 1923, t. 5, p. 259. Cette citation est extraite d'une correspondance conservée aux Archives nationales, $\mathrm{F}^{7} 3438$.

(23) Moser ARnulf, Die französische Emigrantenkolonie in Konstanz während der Revolution (1792-1799), Sigmaringen, Jan Thorbecke, 1975, p. 33-36. Je remercie vivement Karine Rance pour m'avoir communiqué cette référence. 
Lindlar, à l'ouest de Cologne, Jean-Joseph Traizet (1738-1834), curé du diocèse de Soissons, raconte que : "Monsieur le baron de Gheir, qui la veille avait assisté à la messe que j'avais célébrée [chez les carmélites], [se] chargeait de m'offrir le logement, la table et le chauffement dans une maison qui lui appartenait près de l'église, et qu'en outre, $j$ 'aurais tous les jours des intentions de messes avec honoraires pour mon entretien $»^{24}$.

Un document exceptionnel permet de retracer la nature des dépenses d'un prêtre du diocèse de Clermont installé dans les États pontificaux entre 1792 et 1804 . Il s'agit du livre de comptes de Nicolas Marnat (1739-1807), curé de Clémensat ${ }^{25}$. Ce petit livret de 32 feuillets s'intitule : «Depense generale de chaque mois et de chaque année, depuis mon départ de France, le 4 septembre 1792 ». D'une part, l'auteur a reporté sommairement le montant de ses dépenses de chaque mois pour les années 1792 à 1799 ; d'autre part, il a précisé quotidiennement la nature des frais engagés concernant les années 1797 à $1800^{26}$. Cependant, les écritures de cette dernière année semblent avoir été tenues avec beaucoup moins de régularité. Nous avons donc jugé préférable de l'exclure de nos calculs. Avant d'entrer dans le détail des comptes de ce curé auvergnat, il est nécessaire de présenter en quelques mots les modalités de l'accueil mises en place par les autorités pontificales. Pie VI confie à $\mathrm{M}^{\mathrm{gr}}$ Caleppi la charge de répartir les exilés dans des monastères, des couvents ou des maisons religieuses. L'abbé Marnat est installé à Sasso Vivo, abbaye bénédictine du diocèse de Foligno, dans la région de Pérouse. Cette dernière est l'une des quatre grandes circonscriptions (avec Bologne, Ferrare et Viterbe), vers lesquelles sont dirigés les prêtres français. À la fin de l'année 1792, près de 2200 d'entre eux sont présents dans les États pontificaux, dont 130 dans la région de Pérouse ${ }^{27}$. La vie de cette nombreuse communauté est alors réglée selon les injonctions comprises dans la quatrième circulaire, rédigée par $\mathrm{M}^{\mathrm{gr}}$ Caleppi en date du 26 janvier $1793^{28}$. La vie des séculiers comme des réguliers est sévèrement réglementée. Ainsi, les prêtres sont d'une certaine manière placés en résidence

(24) Abbé Louis-Victor PÉcheUR, « Mémoires de l'abbé Traizet, avec une notice sur l'auteur », Bulletin de la société archéologique, historique et scientifique de Soissons, t. 5, 1874, p. 73-158.

(25) Archives départementales du Puy-de-Dôme, F0 107. Il s'agit d'un petit carnet de 11 centimètres de largeur sur 18 centimètres de longueur. Ce document a fait l'objet d'une première transcription en 2007, sous ma direction, par les soins de Jonathan Boudet, étudiant de l'Université Blaise Pascal.

(26) Dans les États pontificaux, l'unité monétaire est le paul, qui comprend dix bayoques. Chaque bayoque vaut dix quattrines. L'écu romain correspond à dix pauls.

(27) R. Picheloup, Les ecclésiastiques français émigrés [...], p. 30-31.

(28) Ibid., p. 180-184. 
surveillée, puisque interdiction leur est faite de quitter le diocèse où ils vivent sans autorisation. Les notes de Nicolas Marnat semblent confirmer la bonne application de ces dispositions car il n'est fait mention d'aucun déplacement en-dehors du diocèse de Foligno. À l'évidence l'administration pontificale se méfie des émigrés. Ainsi, la police surveille attentivement leur correspondance. Sans doute, le curé Marnat dut-il s'engager à observer quelques conditions, afin de satisfaire aux exigences de la vie communautaire. À cet égard, le lyonnais Antoine Vieux donne le détail exact de l'un de ces accords. Reçu le 13 décembre 1792 chez les cordeliers de la Riccardina, près de Bologne, il s'engage d'une part à dire la messe en l'église du couvent les dimanches et fêtes, d'autre part, à assister au chœur avec la communauté, en se conformant aux rites et aux cérémonies de leur ordre et en récitant le bréviaire franciscain. Par ailleurs, il est convenu qu'il partage avec eux l'argent des messes de fondations, tandis que les rétributions des autres messes lui sont assurées ${ }^{29}$.

Les dépenses de l'abbé Marnat peuvent être réparties selon quatre grandes catégories : habillement, nourriture et chauffage, charité et enfin loisirs. Cette répartition peut surprendre dans la mesure où les religieux doivent assurer les frais de bouche et d'hébergement. Ces derniers doivent inclure logiquement le bois de chauffage. Dans le cas présent, il semble évident que ce type de dépenses, une partie tout du moins, est à la charge du pensionnaire des bénédictins olivétains. Ce qui complique également l'analyse des comptes est la présence d'un personnage, du nom d'Ives, pour lequel le scripteur semble prendre en charge une grande partie des besoins alimentaires et vestimentaires. Le 13 février 1797, il note une dépense de dix-neuf bayoques consacrée à l'achat "pour Ives » d'une livre de miel, d'un quart de sucre et de deux œufs frais. Le 9 juin 1799, il paie à « M. Pierre Cruciani Baffo, cordonnier à Foligno », la somme de 18 pauls « pour une paire de souliers pour Ives ». Qui est ce personnage? S'agit-il de l'un de ses confrères? Le seul prêtre portant ce patronyme, est signalé dans le diocèse d'Ancône. Du moins, est-ce son lieu de résidence au moment de son arrivée entre mars 1793 et la fin de l'année suivante $^{30}$. Notre document étant postérieur, peut-être a-t-il ensuite été affecté à Sasso Vivo. Pour des raisons inconnues, sans doute par charité, Nicolas Marnat aurait alors subvenu à ses besoins, pour une part en tout cas.

(29) Jacqueline Tassin, Henri Hours et Émile Poulat, Antoine Vieux. Récit d'émigration, Lyon, Éditions lyonnaises d'Art et d'Histoire, 1991.

(30) R. Picheloup, Les ecclésiastiques français émigrés [...], p. 272. L'auteur livre uniquement son nom, sans préciser son diocèse d'origine. 


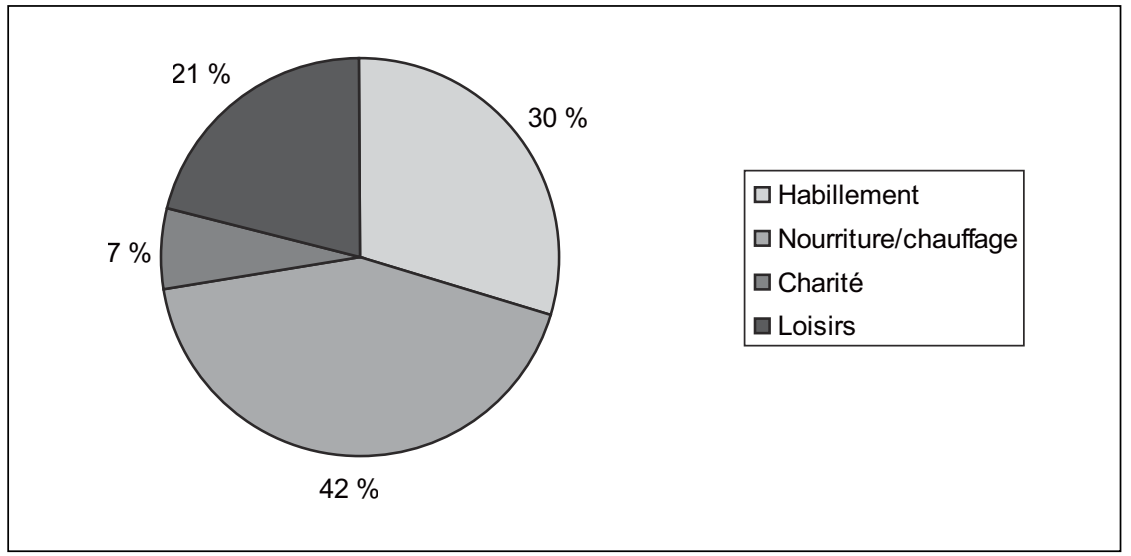

Sans surprise, le graphique de répartition des dépenses pour les années 1797 à 1799 montre le poids important pris par le budget consacré à la nourriture, au chauffage et à l'habillement. Part d'autant plus considérable qu'il s'agit de faire face à l'entretien de deux individus. En bon pasteur, Nicolas Marnat n'oublie pas de pratiquer la charité. La plupart du temps, seule la mention « pauvres » permet de caractériser le geste financier accordé. Ainsi, le 16 avril 1797, trois bayoques sont octroyés à des «pauvres». L'année suivante, ce sont deux Français, bourbonnais et auvergnat, qui sont l'objet de ses sollicitudes : « M. George de Montluçon et Joubert d'Ambert, chirurgiens de l'armée française ». Peut-on déceler ici un quelconque sentiment national ou plus simplement la joie de retrouver des compatriotes? Les dépenses liées aux loisirs ne sont également pas négligeables puisqu'elles totalisent près d'un quart du total. On apprend que l'abbé Marnat est abonné à une gazette, dont il ne livre pas le nom. Il achète également du papier, de même qu'il envoie assez fréquemment des courriers, en France pour l'essentiel. Le 15 avril 1799, il offre même "un gouté au directeur de la poste», sans doute, afin de faciliter ses relations épistolaires! Par ailleurs, notre héros semble faire un usage soutenu de tabac, produit semble-t-il alors de consommation courante, y compris parmi les membres du clergé. Vraisemblablement, il s'agit de tabac à chiquer, dont un autre exilé auvergnat Paul Valeix fait mention à plusieurs reprises ${ }^{31}$. Si l'entretien d'une correspondance ou la

(31) «Le vendredi avant j'avais rencontré en montant à la Poterne Monestier [...]. Il m'assure que c'est mon intérêt de partir [...] ; il prend de mon tabac » ou encore « Revenu, la sentinelle me fait une petite querelle [...]. Je lui fait des excuses sur mon ignorance, je lui donne abondamment de mon bon tabac, le murmure cesse », Abbé Danglard, De Clermont à Genève [...], p. 29 et 56. 
lecture d'un journal représente un lien indispensable avec l'extérieur et permet de tromper l'ennui, il est important de conserver la notion du temps qui s'écoule. Notre curé n'hésite donc pas à dépenser quatre pauls pour faire « décrasser sa montre ».

Les contingences matérielles qui constituent des besoins vitaux ne doivent pas occulter le fait que nos exilés relèvent d'un genre bien particulier. En effet, leur état de prêtre interroge sur la façon dont ils ont pu vivre leur sacerdoce au quotidien. «Peu de personnes oublieront la piété, la conduite irréprochable, la longue et douloureuse patience de ces hommes respectables jetés tout à coup au milieu d'une nation étrangère, différente par sa religion, sa langue, ses mœurs, ses usages. Ils se sont conciliés le respect et la bienveillance de tout le monde par l'uniformité d'une vie remplie de piété et de décence $»^{32}$. Ce jugement élogieux, émis en 1798, est dû au principal ministre anglais William Pitt. Le témoignage de ce champion de la lutte contre la France révolutionnaire plaide, sans ambiguité, en faveur d'un rayonnement sans faille de la spiritualité française. Il brosse le portrait d'un clergé digne et exempt de toute critique. Notre objet n'est pas de juger de la pureté des mœurs de ces clercs. En cette matière, nos documents seraient bien en peine de confirmer ou d'infirmer ces propos. Nous entendons plus modestement interroger ce thème sous l'angle des pratiques et du sentiment religieux. Les exilés essayent, autant que faire se peut, de maintenir une vie liturgique, essentiellement à travers la célébration de la messe ou pour le moins en ayant la possibilité d'y assister. À cet effet, soucieux de disposer d'ornements neufs, Nicolas Marnat commande un amict et trois purificatoires. Il achète également un bréviaire «que Vital Guidi [lui] a porté de Rome », qu'il fait recouvrir de soie et qu'il pare d'un ruban. Le 4 juin 1799, il fait l'achat d' " un Christ de bronze ».

En définitive, tout dépend de chaque situation personnelle. Il est certain que la vie conventuelle, partagée avec leurs hôtes, permet d'entretenir plus facilement cette dimension de la vie spirituelle. Quel regard ces prêtres portent-ils sur leurs épreuves? Le sentiment prédominant est celui d'une divine Providence qui pourvoie à tout, dans les temps malheureux comme dans les plus heureux. Comme l'écrit l'abbé Traizet, à propos d'un inconvénient mineur survenu à Cologne : «Ce n'était qu'une petite épreuve, et la Providence fit bientôt voir qu'elle avait toujours les yeux sur

(32) Citation reproduite dans André Latreille, Jean-Rémy Palanque, Étienne Delaruelle, René RÉmond, Histoire du catholicisme en France, Paris, SPES, 1962, t. 3, p. 122-123. 
nous ». Pour sa part, son confrère normand Pétel, résigné, déclare : « S'il nous eût été donné de nous fixer dans ce beau pays [il évoque alors le village flamand de Stekene] et de passer avec ce bon peuple, le temps de notre exil, nous n'aurions connu ni le malheur, ni l'indigence. Mais Dieu, dans ses décrets, avait arrêté que nous éprouverions l'un et l'autre ». Après tout, ces hommes ont-ils d'autre choix que de s'en remettre à la volonté de celui dont ils sont les serviteurs? Ces références, souvent répétées, à la Providence renforcent l'idée d'une action divine omniprésente. La dimension du "prêtre martyr» n'est donc jamais bien loin, même si elle est rarement exprimée explicitement, si ce n'est parfois par la bouche d'autrui. Ainsi, Vincent Contestin, vicaire de Maillane dans le diocèse d'Arles, rapporte les propos tenus par l'évêque de Nice, $\mathrm{M}^{\mathrm{gr}} \mathrm{de}$ Valpergua, au cours de l'épisode suivant : " [Alors que] nous nous excusions sur nos vêtements indécents et avec lesquels nous nous présentions devant elle [...]. Sa Grandeur ne voulut point recevoir ou écouter nos excuses ». Le prélat s'exclame : «Non, non, Messieurs, n'ayez aucune peine. J'aime à voir des confesseurs de la foi sous un tel costume. Il me présente bien mieux ce qu'ont souffert les martyrs de la primitive Église et m'inspire pour vous encore plus de vénération $»^{33}$. Il n'en est pas moins vrai que les prêtres se sont laissés gagner par les idées du temps. Ainsi, l'anti-monachisme n'est pas absent. L'abbé Pétel ne s'en cache pas lorsqu'il écrit, le 31 décembre 1795, à la suite de l'hospitalité accordée par les capucins de Trente :

« Je n'avais jamais aimé les moines : je fais sans rougir l'aveu de mes torts. Je les avais toujours regardés comme des égoïstes qui, ne s'attachant à personne, ne méritaient pas d'avoir des amis. La barbe des Capucins, quoiqu'elle parût respectable aux yeux de quelques personnes, n'avait pu, elle-même, me guérir de mon préjugé. Cette barbe avait toujours eu, pour moi, quelque chose de repoussant. Aujourd'hui, je reconnais ma faute, et je suis si détrompé que s'il fallait, je baiserais, par reconnaissance, cette barbe qui ne m'avait inspiré que le dégoût ».

Son expérience l'engage donc à revenir sur un jugement trop excessif, même si ces séjours monastiques ne furent pas toujours aussi heureux. Mal reçu par les bénédictins de Fulde, le 8 novembre 1794, Pétel note, non sans une pointe d'ironie, qu'il est conduit en-dehors de l'abbaye,

(33) Abbé Vincent Contestin, « Précis historique de mes voyages pendant la Révolution », Bulletin du comité de l'art chrétien de Nîmes, t. 11, 1923, p. 47-59. 
« dans un bâtiment où quelques couvertures, étendues sur la paille, étaient peut-être là pour rappeler aux bénédictins modernes, la couche des premiers disciples de leur saint fondateur " ! Ces propos se font l'écho d'un siècle de polémiques antireligieuses « qui condamnaient sans nuances les mondes clos, au titre desquels moines et chanoines étaient confondus $»^{34}$. En effet, un seul mot semble devoir caractériser la fonction du prêtre au siècle des Lumières, est-il « utile »? Le terme s'entend ici dans le sens de l'utilité sociale. Le clerc « utile » est celui qui a tout particulièrement en charge une paroisse, loin de tout égoïsme. Le plus souvent, il prend donc les traits du curé de campagne. Ce dernier doit être un homme éclairé, un homme du savoir. Formé au séminaire, possédant une bibliothèque, il est dans la capacité de porter secours à ses contemporains, par exemple en leur faisant part des derniers progrès en matière d'agronomie.

\section{Opinions et observations}

Si le souci le plus pressant est bien celui de se maintenir en vie, le seul fait de prendre le temps nécessaire de l'introspection personnelle afin de rassembler ses réflexions sur son vécu, témoigne à lui seul de la volonté de maintenir une véritable activité intellectuelle. Nos scripteurs font preuve bien souvent d'un fort sens de l'observation qui dépasse la simple curiosité. Pour ce qui concerne le champ politique, les modes de gouvernement des pays traversés n'attirent pas vraiment leur attention. Plus largement, les références aux événements français sont très rares. Ils apparaissent uniquement lorsqu'ils soulèvent l'espoir d'un retour prochain ou, au contraire, quand ils sonnent le glas d'un départ toujours espéré. À la suite des journées des 9 et 10 thermidor de l'an II, Honoré Pétel écrit : « Nous apprîmes, à CEfelt, la fin tragique du tyran Robespierre. Cette nouvelle fit renaître l'espérance dans nos cœurs ». À l'inverse, Antoine Vieux, ayant entrepris de retourner en France, apprend à Turin la nouvelle du coup d'État des 4 et 5 septembre 1797 : «Lorsque, je fus assuré de la vérité de la triste nouvelle, j'étais indécis, si je retournerais en Italie ou si je demeurerais dans le Valais, l'une et l'autre alternative me fatiguait. L'une était périlleuse et pénible et l'autre dispendieuse ».

Le constat de ce faible intérêt peut surprendre de la part de certains, par ailleurs véritables observateurs nés lorsqu'il s'agit de décrire les mœurs ou les cultures. En fait, il semble que le système politique en place

(34) Philippe Bourdin, « Collégiales et chapitres cathédraux au crible de l'opinion et de la Révolution », Annales historiques de la Révolution française, ${ }^{\circ}$ 1, 2003, p. 29-54. 
les préoccupe moins que la garantie de la liberté du culte, et la fin des persécutions à l'encontre du clergé. C'est bien la recherche de stabilité qui leur importe avant toute chose ${ }^{35}$. Engagé sur le chemin du retour vers sa Normandie natale, Pétel, obligé de loger à Vicence, au milieu des troupes françaises, n'éprouve aucune appréhension à se trouver au milieu de ses compatriotes : "D'ailleurs, qu'aurions-nous pu craindre de leur part ? Bonaparte commandait »... Leur sentiment royaliste n'est donc pas un paramètre essentiel. Il apparaît plus succinctement au détour d'une phrase ou d'une observation. Ainsi, à propos de l'exécution de MarieAntoinette, Jean-Joseph Taizet évoque son retour à Bruxelles, « le 16 octobre, jour mémorable où l'on renouvelait en France les horreurs du 21 janvier précédent ». Quant à Pétel, il mentionne Sceaux, « où nous vîmes le château et les jardins qui ont appartenu à l'infortunée reine ». Si les considérations politiques sont peu présentes dans leurs écrits, en revanche, ces prêtres ont parfois à souffrir de manifestations francophobes. Elles proviennent quelquefois de la part de leurs hôtes, en des sentiments qui expriment sans discernement leur rejet de tout ce qui est français. Bien évidemment, dans le cas italien, l'entrée en guerre des armées françaises en 1796 ne fait que renforcer l'hostilité des populations envers tout ce qui porte le nom de Français ${ }^{36}$.

En revanche, le paysage religieux attire plus volontiers les remarques de voyageurs, surtout en pays protestant. Honoré Pétel, alors en Angleterre, écrit que « la privation la plus pénible à notre religion, c'était de ne pas vivre dans un pays catholique ». Plus tard, à Nuremberg, s'étant trouvé momentanément séparé de ses compagnons, il cède à un moment de désespoir en précisant qu'il se trouvait « seul dans une terre étrangère, au milieu des protestants $»$. Il est souvent sans concession lorsqu'il porte un jugement sur les ministres du culte réformé. Reçu à Spalt par un pasteur luthérien, il note que ce dernier « était instruit et parlait assez bien le latin », avant d'ajouter, « ce qui est rare parmi les ministres protestants ». Surpris de trouver dans un temple, des « images de la Sainte Vierge et des

(35) Les débats politiques sont surtout l'apanage du haut clergé. Voir Yann Fauchois, « Les émigrés français et le royalisme pendant la Révolution française », dans François LeBRUN et Roger Dupuy (dir.), Les résistances à la Révolution, Paris, Imago, 1987, p. 386-395 et Bernard Plongeron, «Soumission aux loi de la République ? La fin d'un consensus politique parmi les évêques émigrés (1795-1802) », dans Jean-Clément Martin (dir.), Religion et Révolution, Paris, Anthropos, 1994, p. $171-181$.

(36) À ce sujet, voir la contribution de Paul Chopelin, « Des Loups déguisés en agneaux ? L'accueil des prêtres constitutionnels émigrés dans l'État pontifical (1792-1799) », Annales historiques de la Révolution française, $\mathrm{n}^{\circ} 341,2005$, p. 85-109. 
Saints », il s'entend répondre de la bouche du pasteur que « les luthériens ne ressemblent pas aux calvinistes, qui ne se sont signalés que par la destruction et les ravages ». Sous la plume d'un prêtre catholique, cet épisode n'est pas tout à fait anodin, lorsqu'on songe que l'immense majorité des réformés français est rattachée à l'Église de Calvin... Lors de son séjour en Gueldre prussienne, il déplore qu'habituellement « la principale église » appartienne aux protestants, tandis que les catholiques « quoique infiniment plus nombreux, n'ont pour l'exercice de leur culte, que des bâtiments couverts de paille ». Mais, il s'empresse d'ajouter combien l'agencement intérieur de ces pâles édifices pallie largement ce défaut : «Ces humbles chaumières sont parfaitement décorées. Tout y annonce la piété généreuse des catholiques qui s'y réunissent : tableaux, peintures, tribunes, orgues, vases précieux, riches ornements, concours immense de peuple, tout marque le respect de ces chrétiens, fidèles pour la majesté du Dieu qu'ils adorent [...] ». On pressent ici le désir de l'auteur de rendre hommage à des coreligionnaires restés fidèles à leur foi, dans un environnement religieux pluriel. Sa description d'un peuple chrétien tout imprégné de religiosité, même si elle est sublimée, renvoie également en creux à une situation française qui ne correspond plus à cette vision idyllique. L'observateur ne peut que le regretter... Ces assertions, parfois sans concessions envers les réformés, n'empêchent pas Pétel de porter un jugement favorable sur ces pays protestants : "C'est, cependant, cette nation [1'Angleterre] qui nous a reçus avec l'humanité la plus généreuse et la plus compatissante ». Plus loin, il précise : « Le peuple qui, quelques mois auparavant, aurait eu du plaisir à couvrir d'insultes un prêtre romain, nous vit arriver avec l'intérêt que commande le respect. Une seule chose leur faisait peine, c'était disaient-ils, que nous avions abandonné nos femmes et nos enfants: "Eh! Pourquoi, ajoutaient-ils, ne les avez-vous pas conduits avec vous ?". Il nous fallut quelque temps pour les détromper, car leurs ministres ayant leurs femmes, ils croyaient fermement que nous étions mariés comme eux ». Quel lent et difficile apprentissage commun que celui de l'altérité !

Plus largement, les mœurs et les pratiques culturelles des autochtones ne manquent pas de solliciter la plume de ces expatriés, parfois surpris par les coutumes et les usages locaux, toujours curieux d'en savoir plus. La façon de s'habiller attire facilement les commentaires, tel que celui de Pétel, à propos des femmes de la région de Lucerne : "Leur costume nous parut singulier. Leurs jupes sont attachées sous le sein et descendent, tout au plus, jusqu'au gras de la jambe. Elles ont la tête nue, comme les Allemandes et les Italiennes, mais leurs cheveux, au lieu d'être 
liés sur leurs têtes, tombent en deux tresses sur les épaules, et quand ils ne sont pas assez longs pour descendre jusqu'aux talons, elles y ajoutent des rubans ». Les villes traversées ne manquent pas de soulever les remarques les plus diverses. Comparons les récits concernant quelques cités d'Italie du nord, en l'occurrence ceux de Jean-Claude Souzy-Thomas (17601753), vicaire de Saint-Loup dans le diocèse de Lyon $^{37}$, d'Antoine Boussac (1765-1854), vicaire à Moulins dans le diocèse d'Autun ${ }^{38}$ et d'Antoine Vieux, déjà évoqué. Des trois descriptions, celle d'Antoine Vieux est toujours la plus riche. Passant tous les trois à Verceil [Vercelli], Antoine Boussac, généralement le moins bavard, indique simplement qu'il s'agit d' « une ville commerçante et assez gentille. Sa population est d'environ huit mille âmes ». A. Vieux et J.-C. Souzy s'accordent pour dire que la ville est « bien bâtie ». Alors que le second signale assez rapidement la cathédrale, ainsi que les tombeaux de saint André ou de saint Eusèbe, le premier s'attarde dans une description plus avancée, notamment en évoquant l'église Saint-Vincent. La traversée de Plaisance [Piacenza] recueille des sentiments beaucoup plus mitigés. Pour Boussac, l'affaire est très vite entendue : "Ville bâtie dans le goût moderne et très agréable. Sa population est d'environ quarante mille âmes ; la lassitude nous empêchant d'en admirer les beautés, nous en partîmes ». Pour leur part, Souzy et Vieux ont des avis parfois assez divergents. Si tous les deux reconnaissent que la ville dégage un certain charme, étant « agréablement située sur les bords du Pô », selon les termes de Souzy ; ce dernier indique également qu'on voit sur la place du gouvernement « deux statues équestres qui n'ont rien de beau ». Ce jugement n'est pas partagé par son confrère qui estime que ces deux statues équestres offrent au regard des chevaux qui « sont faits avec tant d'art qu'ils semblent lancer le feu par la bouche et les narines ». Bologne enfin est une cité qui attire largement l'attention de nos visiteurs. Pour Boussac, elle est « après Rome », la ville « la plus belle et la plus florissante de toutes celles qui appartiennent au pape », alors que de l'aveu d'A. Vieux, « il faudrait un gros volume pour [en] noter les particularités et les beautés ». Souzy s'avère beaucoup plus circonspect dans ses commentaires. Ses critiques, comme souvent, sont parfois assez sévères. Par exemple, il note qu' « il n'y a qu'une place devant le palais public et encore

(37) Gilbert GaRdEs, "Journal de voyage d'un prêtre réfractaire émigré en Italie (1792-1797)», Bulletin de la Diana, t. 64, 2005, p. 74-100.

(38) Abbé Joseph CLÉMent, Itinéraire d'un prêtre bourbonnais " déporté " pendant la Révolution française. Journal de voyage de M. l'Abbé Boussac, Moulins, H. Durond, 1899. 
elle est fort petite : le Neptune en bronze qui est au milieu est digne d'être vu quoique indécent $» !$

Sur le plan économique, ce sont essentiellement les activités agricoles qui attirent l'attention des scripteurs, « cet art, le premier et le plus précieux de tous », comme l'écrit le vicaire normand, Louis Nicolas Lemaitre (1762-1829) ${ }^{39}$. Cet aspect ayant fait l'objet d'une contribution récente, nous nous permettons de nous référer à ses principales conclusions. Bernard Bodinier souligne l'enthousiasme des scripteurs face à la grande variété des cultures, en émettant toutefois certaines réserves, notamment pour ce qui concerne l'élevage allemand. Les méthodes de travail reçoivent un accueil mitigé, tandis que l'utilisation de certains engrais, en l'occurrence les excréments humains, les laissent parfois dubitatifs. Ces descriptions n'excluent donc pas « un regard critique, voire contradictoire $»$. Par ailleurs, s'ils négligent certains aspects, tels que l'habitat rural ou le système de propriété, «ils manifestent un intérêt certain pour les techniques ou méthodes qu'ils découvrent $\gg$. De fait, l'exil a favorisé les échanges avec des civilisations jusque là peu connues, voire totalement ignorées. Ces contacts sont difficiles à mesurer. Seules quelques indications permettent de les subodorer. L'éditeur du journal de l'abbé Peccard indique que celui-ci «aimait dans l'intimité conter des anecdotes relatives à son exil et chanter des vieux cantiques espagnols ». Au cours de l'été 1817, il entreprend même un voyage sur les lieux de son ancien séjour. Enfin, pour terminer, il convient de signaler que l'exil ne fut pas toujours un séjour de larmes et de tristesse. Les situations vécues sont souvent l'occasion d'envisager, avec un certain recul, des inconvénients mineurs ${ }^{40}$. En cela, ces écrits témoignent avant tout d'une aventure humaine, faite de nombreuses difficultés, mais également de petites joies. Jean-Claude Souzy-Thomas s'en fait l'écho à plusieurs reprises. Obligé de faire raccommoder ses souliers, le cordonnier lui demande 18 sols pour son ouvrage : «Je n'en avais que 17 , ce qui nous fit beaucoup rire ». Dans une auberge, alors que le souper arrive sur la table : « Nous rîmes

(39) Cité par Bernard Bodinier, « Prêtres normands exilés pendant la Révolution et agriculture européenne », dans Florent Quellier et Georges Provost (dir.), Du ciel à la terre. Clergé et agriculture $X V I^{e}-X I X^{e}$ siècles, Rennes, PUR, 2008, p. 127-142. Cet auteur est sans doute le premier à avoir traité ces écrits de façon sérielle, afin d'éclairer une thématique bien particulière. Par ailleurs, nous le remercions pour nous avoir communiqué des références précieuses concernant ces prêtres normands.

(40) B. Plongeron note également que les « exilés savent plaisanter de leurs propres misères », «Église et Révolution, d'après les prêtres émigrés à Rome et à Londres (1792-1802) » $[\ldots]$, p. 302. 
beaucoup d'un plat énorme par sa grandeur dans lequel on servit une petite soupe $»$.

Au total, ces écrits témoignent d'aventures personnelles. En cela, le monde des émigrés apparaît dans toute sa diversité et sa complexité. Cependant, ils permettent aussi d'éclairer le devenir d'un groupe en particulier. Longtemps considérés, soit comme des martyrs, soit comme de dangereux contre-révolutionnaires, leurs écrits dévoilent tout d'abord des hommes qui ressentent des émotions souvent contradictoires, mais révélatrices de la condition humaine. En effet, ils passent parfois de l'obstination la plus exacerbée, pétrie parfois de quelques illusions, au découragement et à la lassitude les plus terribles. Pour l'essentiel, ils ont cherché surtout à survivre dans un environnement étranger et parfois hostile. En cela, conjointement avec l'expérience de la peur, ils ont connu différents types d'épreuves : celui de l'espace et des éléments, celui de l'hospitalité. Mais, l'exil est également le lieu de la rencontre. En définitive, nous espérons avoir montré que ces témoignages sont plus que « des relations souvent attentives, parfois pittoresques, puériles à l'occasion, de gîtes divers, de relais et d'étapes, de raisons justifiées de gratitude ou de mauvaise humeur $»^{41}$.

Stéphane GomIS

Centre d'histoire « Espaces et cultures » Université Blaise-Pascal (Clermont II)

4, rue Ledru

63057 Clermont-Ferrand Cedex 1 Stephane.GOMIS@univ-bpclermont.fr

(41) Fernand Baldensperger, Le mouvement des idées dans l'Émigration française (1789-1815), Paris, Plon, 1925, t. 1, p. 130. 